\title{
Bidimensional planar micro-optics for optochemical absorbance sensing
}

\author{
I. Garcés \\ Departamento Ingeniería Electrónica y Comunicaciones, Universidad de Zaragoza, María de Luna, 3. 50015 Zaragoza, Spain \\ F. Villuendas and J. Subías \\ Departamento Física Aplicada, Universidad de Zaragoza, 50009 Zaragoza, Spain \\ J. Alonso and M. del Valle \\ Departamento Química Analítica, Universidad Autónoma de Barcelona, 08193 Bellaterra, Spain \\ C. Domínguez and E. Bartolomé \\ Centro Nacional de Microelectrónica, 08193 Bellaterra, Spain
}

Received September 22, 1997

\begin{abstract}
A new approach for developing optochemical absorbance sensors is presented. The method is based on a planar micro-optic circuit in which an optochemically active membrane that responds to selective compounds is deposited in the device, yielding a part of the guiding planar structure. In this way the optical field is confined in the direction transverse to the substrate and controlled in the lateral direction by means of planar micro-optics components. High sensitivity of the device can be easily obtained because of the relatively long light paths through the membrane, and the response time is low because the analyte has to diffuse through a several-micrometer-thin membrane. Experimental results of measurements of the concentration of potassium are also presented to verify the possibilities of these devices as specific absorbance sensors. (C) 1998 Optical Society of America

OCIS codes: $130.0130,230.0230,130.6010,130.3120,230.7370$.
\end{abstract}

Optical fiber chemical sensors can play an important role in chemical detection because they combine the characteristics and advantages of optochemical detection with the possibility of developing miniaturized compact devices that can be included in standard analytical chemical and medical labs as well as in control of industrial chemical processes. Optochemical sensors based on the change of the imaginary part of the refractive index, i.e., optical absorbance sensors, have been widely studied and are usually based on the interaction of the evanescent field of guided light with the analytes ${ }^{1}$ or on bulk discrete components. ${ }^{2}$ In the first case long interaction distances or a large field out of the waveguide is required, which makes the response of the sensors very dependent on variations of the real part of the refractive index of the solution containing the analyte. In the other case the devices have low sensitivity and dynamic range and are often disturbed by the optical characteristics of these solutions. However, optical absorbance sensors can provide a suitable approach for an important range of applications owing to the possibility of designing specific devices to identify different compounds. The specificity and ease of use of optochemical absorbance sensors have been improved by the development of chemical membranes in which specific compounds are immobilized in a polymeric matrix (optode membranes). ${ }^{3}$ These membranes are formulated with a polymer support, normally poly(vinyl chloride), and an ionophore, a special compound that chemically recognizes the target species, which is dissolved in a suitable organic solvent or plasticizer. The ionophore acts here as a selective carrier for a particular chemical species. If the latter is charged, for the extraction process to be feasible a coextraction step is needed to maintain electrical neutrality inside the organic phase. So the membrane is designed in such a way that the proton $\left(\mathrm{H}^{+}\right)$is the coextracted ion and another special compound, the chromoionophore, interacts with this ion, altering its spectrum, and accordingly changing the absorption of the light propagating through the membrane.

In this sense a new approach for developing optochemical absorbance sensors is presented in this Letter. This approach is based on a planar micro-optics circuit in which an optochemically active membrane is deposited upon the device, constituting a part of the guiding planar structure. So light propagating through the planar circuit is confined in the direction transverse to the substrate by the membrane and controlled in the lateral direction by planar bidimensional micro-optical components.

A first, simple prototype of the sensor design, shown in Fig. 1, is composed of a single-mode channelintegrated optics waveguide that is coupled to a single-mode fiber and acts as an input waveguide, a free propagation region between waveguides in which the membrane is placed, and a wider multimode channel guide that is coupled to a multimode fiber and acts as an output waveguide. The input and output waveguides consist of $10-$ and $50-\mu \mathrm{m}$-wide rib antiresonant reflecting optical waveguide (ARROW) structures, ${ }^{4}$ used because of their tolerance fabrication parameters and the height of the core of singlemode waveguides $(4 \mu \mathrm{m}),{ }^{5}$ which allows the use of 


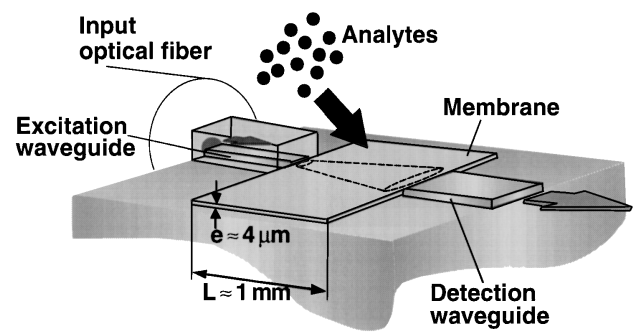

Fig. 1. Configuration of the proposed micro-optical planar sensor structure, including the input optical fiber, excitation waveguide, membrane region, and detection waveguide.

achievable thick membranes and matches the size of the input optical fiber. The input waveguide must be embedded, that is, shielded from the external medium so that disturbances in the modal distribution, and consequently in the optical power launched into the membrane, are avoided. Light coupled from the input waveguide into the membrane propagates through it, is confined in the direction perpendicular to the substrate, and is diffracted in the lateral direction. This lateral diffraction implies a broadening of the input beam, which determines the length of the free propagation region as a compromise between sensitivity and dynamic range and low insertion losses in the output waveguide coupling. Simulations of the device show that insertion losses of less than $1 \mathrm{~dB}$ are achievable for path lengths of $1 \mathrm{~mm}$, taking into account the usual refractive-index range of the membrane (1.40-1.50) and assuming that the optical fields propagating through the different parts of the circuit match each other in the transverse direction. Disturbances at this point would imply additional insertion losses. Light that propagates through the membrane is absorbed by immobilized chromoionophores as a function of the analyte concentration, which has to diffuse in the direction transverse to the membrane, as is depicted in Fig. 1.

Several prototypes were fabricated with silica-onsilicon complementary metal-oxide semiconductorcompatible processes, and the multilayer ARROW structure was designed to be polarization independent at the $670-\mathrm{nm}$ wavelength. ${ }^{5}$ The rib structure that is necessary for guiding in the lateral direction, and the free propagation region were fabricated over this multilayer structure by successive application of reactive ion etching. Top and front views of the fabricated sensor are depicted in Figs. 2a and 2b, respectively; Fig. 2a shows the input and output waveguides and the free propagation membrane region. At each end of the device, two continuous waveguides similar to the input and output waveguides were constructed to verify the behavior of the rib ARROW structures.

The optically active medium used in the device, an optode membrane, is responsible for the detection principle of the sensor. This membrane consists of a polymeric matrix made of poly(vinyl chloride) and a lipophilic plasticizer [(bis(2-ethylexyl) sebacate] in which several chemical and optochemically active components are dissolved. Specifically, a chromoionophore that changes its absorption bands at the operating wavelength of the device-specific ionophore for the ion to be analyzed, and a diffusion-process activator should be utilized. The components used depend on the specific application, which in our case is potassium-concentration measurements, because of the well-established procedure for recognizing potassium and its potential usefulness in clinical analysis. Valinomycin is the specific ionophore used for recognizing potassium because of its very small relative sensitivity $\left(<10^{-3}\right)$ to other cations; lipophilized Nile Blue, which changes its absorption bands in the range of $670 \mathrm{~nm}$, is used as a chromoionophore, and potassium tetrakis[3.5-bis(trifluoromethyl)phenyl] borate is the process activator.

The optical source used for the optical characterization of the devices is a 20-nm spectral-width LED operating at $670 \mathrm{~nm}$ that couples $1 \mu \mathrm{W}$ of optical power in a single-mode fiber at this wavelength and is stabilized by a monitor photodiode. This input fiber is a $4-\mu \mathrm{m}-$ diameter single-mode fiber that matches the vertical size of the excitation waveguide core, thus reducing insertion losses. Coupling of light from the optical fiber to the excitation waveguide, and from the detection waveguide to the detection optical fiber, is achieved by means of a positioning system that is based on a set of high-precision motion stages upon which the fibers and the sensor are placed. The detection optical fiber is a $50-\mu \mathrm{m}$-diameter multimode fiber that is coupled to a silicon photodiode by means of standard optical fiber connectors. The guiding characteristics of the waveguides were measured, and attenuation values of $\sim 0.3 \mathrm{~dB} / \mathrm{cm}$ in the spectral range of 600 to $800 \mathrm{~nm}$, low polarization dependence, and insertion losses of $1.5 \mathrm{~dB}$ between a single-mode fiber and the input waveguide were obtained. These features give rise to less than 2 -dB insertion losses from fiber to fiber at the $670-\mathrm{nm}$ wavelength, as depicted in Fig. 3.

The sensor insertion losses were $18 \mathrm{~dB}$ at the operating wavelength of $670 \mathrm{~nm}$ for a 1-mm-long
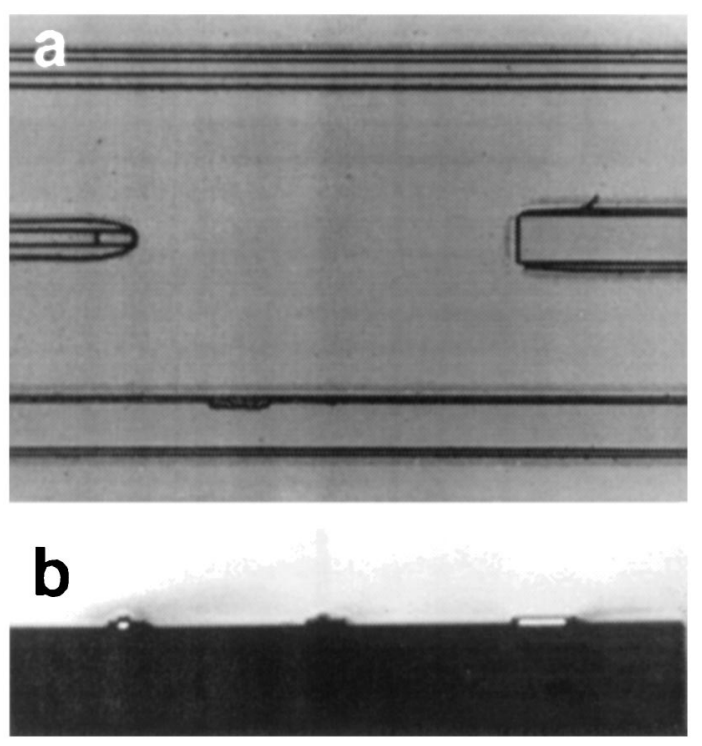

Fig. 2. Photographs of the fabricated sensor: a, upper view of the waveguides and the free propagation region; $\mathrm{b}$, front view of the waveguides. 


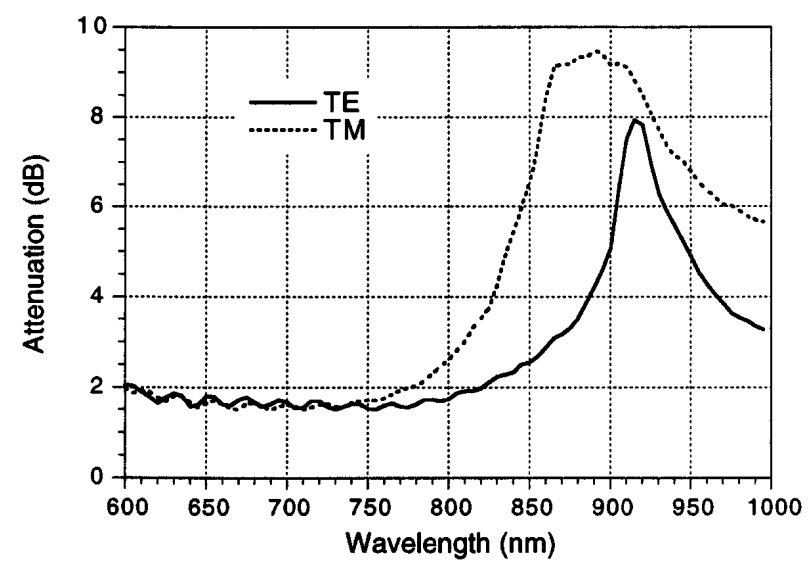

Fig. 3. Experimental attenuation response of a 1-cm-long rib ARROW waveguide as a function of wavelength for both TE and TM polarizations.

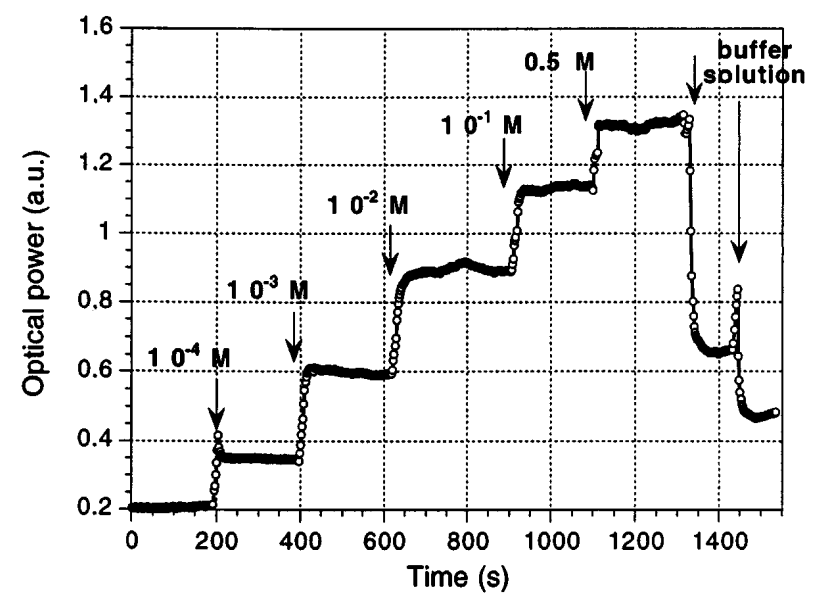

Fig. 4. Measured response of the sensor for several concentrations of potassium.

free propagation region: Scattering and absorption losses owing to the polymeric matrix of the membrane were $6 \mathrm{~dB}$, attenuation losses owing to the residual absorption coefficient of the chromoionophore were $4 \mathrm{~dB}$, the intrinsic attenuation of the waveguides was $2 \mathrm{~dB}$, and diffraction-based calculated losses were $1 \mathrm{~dB}$. Therefore an additional $5 \mathrm{~dB}$ should be attributed to fabrication defects owing to membrane inhomogeneities and mainly to deficiencies in the output and input faces of the excitation and detection waveguides. With these insertion losses, a dynamic range of more than $30 \mathrm{~dB}$, limited by the signal/noise ratio in the measured optical power, was obtained in the device.

The possibilities of bidimensional micro-optics-based sensors were tested by several measurements of potassium-ion concentration with different devices and membranes. The solution to be measured consisted of potassium chloride diluted in a 0.5-M magnesium acetate buffer solution with a concentrations of $10^{-4}$ to $0.5 \mathrm{M}$. The buffer solution was used to stabilize the $\mathrm{pH}$ of the membrane at a fixed value of 5.5. The $\mathrm{pH}$ value determines the quantity of available protons and, accordingly, the optimum range of $\mathrm{K}^{+}$concentration for the sensor response.

The experimental procedure consisted of placing drops of different concentrations of the solution over the active area of the device and measuring the optical power once every second. Results obtained for a specific device are shown in Fig. 4, in which optical power variations as a function of time are measured for several $\mathrm{K}^{+}$concentrations. In any case an achievable dynamic range of $\sim 30 \mathrm{~dB}$ in the measured optical power was observed, which represents 3 absorbance units for sensors with 1-mm-long free propagation region.

Figure 4 also shows the response time of the device, which is as short as a few seconds, which corresponds to the time for diffusion through thin membranes. The membrane behavior was stable over several days, and this time would be greatly increased if a photocuring membrane were used. Moreover, some instabilities appear in Fig. 4 owing to inhomogeneous diffusion processes and mixing of different concentrations because of the difficulty of cleaning the membrane in the experimental procedure used. However, these kinds of inconvenience can be easily overcome with a flow-cell configuration.

In summary, the approach that has been proposed may provide a new way to solve some of the problems of the present optochemical absorbance sensors. In particular, short diffusion time can be achieved since the membrane is only a few micrometers thick, and high dynamic range and sensitivity are achieved with the extended optical path inside the membrane. Interferences owing to the optical characteristics of the solution are minimized because the light does not propagate through the external medium. Therefore optochemical absorbance sensors with high sensitivity and dynamic range, fast response, and specificity can be developed. The planar micro-optic circuit and the active membrane represent the optical basis of the sensor, and thus the optical field is confined in the direction perpendicular to the substrate and is controlled in the lateral direction by a suitable integrated optical circuit design. Furthermore, optimized designs for specific applications can be developed if proper refractive or reflexive bidimensional planar components, including different types of waveguide, are used. Applications with several propagation lengths through the membrane, different sensitivities and dynamic ranges of the device, optode configurations, referencesignal or multiple specific sensors in a chip can be implemented by use of suitable bidimensional circuits.

This research was financially supported by the Comision Interministerial de Ciencia Y Tecnologia (project number TIC93-0525).

\section{References}

1. L. Yang and S. Saavedra, Anal. Chem. 67, 1307 (1995).

2. E. Bakker, M. Lerchi, T. Rosatzin, B. Rusterholz, and W. Simon, Anal. Chim. Acta 278, 211 (1993).

3. U. Spichiger, W. Simon, E. Bakker, M. Lerchi, P. Buhlmann, J. Haug, M. Kuratli, S. Ozawa, and S. West, Sensors Actuators B 11, 1 (1993).

4. M. A. Duguay, Y. Kokubun, T. L. Koch, and L. Pfeiffer, Appl. Phys. Lett. 49, 13 (1986).

5. I. Garcés, F. Villuendas, J. Vallés, C. Domínguez, and M. Moreno, IEEE J. Lightwave Technol. 14, 798 (1996). 